\title{
The dispersionless Veselov-Novikov equation: symmetries, exact solutions, and conservation laws
}

\author{
Oleg I. Morozov ${ }^{1}$ (1) Jen-Hsu Chang ${ }^{2}$ \\ Received: 8 February 2021 / Accepted: 9 June 2021 / Published online: 18 June 2021 \\ (c) The Author(s) 2021
}

\begin{abstract}
We study symmetries, invariant solutions, and conservation laws for the dispersionless Veselov-Novikov equation. The emphasis is placed on cases when the ODEs involved in description of the invariant solutions are integrable by quadratures. Then we find some non-invariant solutions, in particular, solutions that are polynomials of an arbitrary degree $N \geq 3$ with respect to the spatial variables. Finally we compute all conservation laws that are associated to cosymmetries of second order.
\end{abstract}

Keywords Dispersionless Veselov-Novikov equation · Symmetry · Exact solution · Conservation law

Mathematics Subject Classification $35 \mathrm{G} 20 \cdot 35 \mathrm{Q} 60 \cdot 17 \mathrm{~B} 50 \cdot 22 \mathrm{E} 70$

\section{Introduction}

We consider the dispersionless Veselov-Novikov equation (dVN) [11] written in the form

$$
u_{t x y}=\left(u_{x x} u_{x y}\right)_{x}+\left(u_{x y} u_{y y}\right)_{y} .
$$

This equation describes the propagation of the high frequency electromagnetic waves in certain nonlinear media, see [12] and references therein. Nontrivial $t$-independent

Oleg I. Morozov

morozov@agh.edu.pl

Jen-Hsu Chang

jhchang@ndu.edu.tw

1 Faculty of Applied Mathematics, AGH University of Science and Technology, Al. Mickiewicza 30, 30-059 Kraków, Poland

2 Graduate School of National Defense, National Defense University, Dashi District, Tao-Yuan City 33551, Taiwan 
solutions of $\mathrm{dVN}$ are related to the problem of existence of the first integrals for the geodesic or magnetic geodesic flows on a two-torus, [23]. Equation (1) is the dispersionless reduction of the Nizhnik-Veselov-Novikov equation [17,24]. The Lax representation [21]

$$
\left\{\begin{array}{l}
q_{t}=\frac{1}{3}\left(q_{x}^{3}-\frac{u_{x y}^{3}}{q_{x}^{3}}\right)+u_{x x} q_{x}-\frac{u_{x y} u_{y y}}{q_{x}}, \\
q_{y}=-\frac{u_{x y}}{q_{x}} .
\end{array}\right.
$$

of $\mathrm{dVN}$ is the dispersionless reduction of the Lax representation of the NizhnikVeselov-Novikov equation. In [2] the Lax representation (2) was used to construct two-dimensional reductions of $\mathrm{dVN}$.

In the present paper we study exact solutions and conservation laws of $d V N$. We find the contact symmetry algebra and the explicit form for the transformations from the contact symmetry pseudogroup of $\mathrm{dVN}$. Then we employ the pseudogroup to find the optimal system of one-dimensional subalgebras of the symmetry algebra. We factorize dVN with respect to the symmetries from the optimal system and obtain two-dimensional partial differential equations (PDEs) (8) and (50) for the invariant solutions as well as their Lax representations. Then we find the symmetry algebras and their optimal systems of one-dimensional subalgebras for equations (8) and (50). The factorization with respect to the subalgebras provide the collection of ordinary differential equations (ODEs) that describe invariant solutions to (8) and (50). We find some cases when the obtained ODEs are integrable by quadratures, thus providing exact solutions for dVN. Further, we study solutions that are not invariant with respect to contact symmetries. In particular, we find a class of solutions to $\mathrm{dVN}$ that are polynomials in $x$ and $y$ of arbitrary degree. Finally we find the whole set of conservation laws that are associated to cosymmetries defined on the second order jets.

\section{Preliminaries}

The presentation in this section closely follows $[13,15,25]$. Let $\pi: \mathbb{R}^{n} \times \mathbb{R} \rightarrow \mathbb{R}^{n}$, $\pi:\left(x^{1}, \ldots, x^{n}, u\right) \mapsto\left(x^{1}, \ldots, x^{n}\right)$, be a trivial bundle, and $J^{\infty}(\pi)$ be the bundle of its jets of infinite order. The local coordinates on $J^{\infty}(\pi)$ are $\left(x^{i}, u, u_{I}\right)$, where $I=\left(i_{1}, \ldots, i_{n}\right)$ are multi-indices, and for every local section $f: \mathbb{R}^{n} \rightarrow \mathbb{R}^{n} \times \mathbb{R}$ of $\pi$ the corresponding infinite jet $j_{\infty}(f)$ is a section $j_{\infty}(f): \mathbb{R}^{n} \rightarrow J^{\infty}(\pi)$ such that $u_{I}\left(j_{\infty}(f)\right)=\frac{\partial^{\# I} f}{\partial x^{I}}=\frac{\partial^{i_{1}+\cdots+i_{n}} f}{\left(\partial x^{1}\right)^{i_{1}} \ldots\left(\partial x^{n}\right)^{i_{n}}}$. We put $u=u_{(0, \ldots, 0)}$. Also, we will simplify notation in the following way, e.g., in the case of $n=3$ : we denote $x^{1}=t$, $x^{2}=x, x^{3}=y$, and $u_{(i, j, k)}=u_{t \ldots t x \ldots x y \ldots y}$ with $i$ times $t, j$ times $x$, and $k$ times $y$.

The vector fields

$$
D_{x^{k}}=\frac{\partial}{\partial x^{k}}+\sum_{\# I \geq 0} u_{I+1_{k}} \frac{\partial}{\partial u_{I}}, \quad k \in\{1, \ldots, n\},
$$


$\left(i_{1}, \ldots, i_{k}, \ldots, i_{n}\right)+1_{k}=\left(i_{1}, \ldots, i_{k}+1, \ldots, i_{n}\right)$, are called total derivatives. They commute everywhere on $J^{\infty}(\pi)$ and are annihilated by the ideal of contact forms $\left\langle d u_{I}-\sum_{k=1}^{n} u_{I+1_{k}} d x^{k} \mid \# I \geq 0\right\rangle$.

The evolutionary vector field associated to an arbitrary smooth function $\varphi: J^{\infty}(\pi)$ $\rightarrow \mathbb{R}$ is defined as

$$
\mathbf{E}_{\varphi}=\sum_{\# I \geq 0} D_{I}(\varphi) \frac{\partial}{\partial u_{I}}
$$

with $D_{I}=D_{\left(i_{1}, \ldots i_{n}\right)}=D_{x^{1}}^{i_{1}} \circ \cdots \circ D_{x^{n}}^{i_{n}}$.

A PDE $F\left(x^{i}, u_{I}\right)=0$ of order $s \geq 1$ with $\# I \leq s$ defines the submanifold $\mathcal{E}=$ $\left\{\left(x^{i}, u_{I}\right) \in J^{\infty}(\pi) \mid D_{K}\left(F\left(x^{i}, u_{I}\right)\right)=0, \# K \geq 0\right\}$ in $J^{\infty}(\pi)$.

A function $\varphi: J^{\infty}(\pi) \rightarrow \mathbb{R}$ is called a (generator of an infinitesimal) symmetry of equation $\mathcal{E}$ when $\mathbf{E}_{\varphi}(F)=0$ on $\mathcal{E}$. The symmetry $\varphi$ is a solution to the defining system

$$
\ell_{\varepsilon}(\varphi)=0,
$$

where $\ell_{\varepsilon}=\left.\ell_{F}\right|_{\mathcal{E}}$ with the differential operator

$$
\ell_{F}=\sum_{\# I \geq 0} \frac{\partial F}{\partial u_{I}} D_{I} .
$$

The symmetry algebra $\operatorname{Sym}(\mathcal{E})$ of equation $\mathcal{E}$ is the linear space of solutions to (3) endowed with the structure of a Lie algebra over $\mathbb{R}$ by the Jacobi bracket $\{\varphi, \psi\}=$ $\mathbf{E}_{\varphi}(\psi)-\mathbf{E}_{\psi}(\varphi)$. The algebra of contact symmetries $\operatorname{Sym}_{0}(\mathcal{E})$ is the Lie subalgebra of $\operatorname{Sym}(\mathcal{E})$ defined as $\operatorname{Sym}(\mathcal{E}) \cap C^{\infty}\left(J^{1}(\pi)\right)$. The point symmetries are the contact symmetries whose generators are polynomials in $u_{x^{i}}$ of degree 1 .

The contact symmetry pseudogroup of a PDE $\mathcal{E} \subset J^{\infty}(\pi)$ is the collection of all the local diffeomorphisms $\Gamma_{\infty}: J^{\infty}(\pi) \rightarrow J^{\infty}(\pi)$ that preserve the submanifold $\mathcal{E}$ as well as the ideal of contact forms.

Let $\phi$ be a symmetry of $\mathcal{E}$, then the $\phi$-invariant solution of $\mathcal{E}$ is the solution of the compatible system $F=0, \phi=0$.

For a PDE $\mathcal{E}$ in three independent variables $t, x, y$ a conservation law, $[18, \S 4.3]$, [25, Ch. 5], is a horizontal two-form

$$
\Omega=P_{1} d x \wedge d y+P_{2} d y \wedge d t+P_{3} d t \wedge d x,
$$

closed with respect to the horizontal differential $d_{h}$, which means that

$$
d_{h} \Omega=\left(D_{t}\left(P_{1}\right)+D_{x}\left(P_{2}\right)+D_{y}\left(P_{3}\right)\right) d t \wedge d x \wedge d y=0
$$

on $\mathcal{E}$. Functions $P_{i}$ are smooth functions on $\mathcal{E}$. The conservation law is referred to as a trivial conservation law when there exists a horizontal one-form $\omega$ such that $\Omega=d_{h} \omega$. Nontrivial conservation laws are associated with cosymmetries of equation 
$\mathcal{E}$, see discussion in $\left[14\right.$, Ch. 1]. Let $\tilde{P}_{i}$ be arbitrary extensions of $P_{i}$ on $J^{\infty}(\pi)$, then for $\tilde{\Omega}=\tilde{P}_{1} d x \wedge d y+\tilde{P}_{2} d y \wedge d t+\tilde{P}_{3} d t \wedge d x$ there holds $d_{h} \tilde{\Omega}=\psi \cdot F d t \wedge d x \wedge d y$ for some function $\psi$ on $J^{\infty}(\pi)$. The restriction $\left.\psi\right|_{\mathcal{E}}$ depends on $\Omega$ only and is called the generating function or characteristic of the conservation law $\Omega$. The conservation law is trivial if and only if its generating function vanishes. Generating functions are solutions to equation

$$
\ell_{\mathcal{E}}^{*}(\psi)=0
$$

with $\ell_{\mathcal{E}}^{*}=\left.\ell_{F}^{*}\right|_{\mathcal{E}}$, where the adjoint operator to $\ell_{F}$ is

$$
\ell_{F}^{*}=\sum_{\# I \geq 0}(-1)^{|I|} D_{I} \circ \frac{\partial F}{\partial u_{I}} .
$$

A solution of (4) is referred to as a cosymmerty of equation $\mathcal{E}$.

\section{Symmetries of dVN}

\subsection{The symmetry algebra}

Direct computations ${ }^{1}$ show that the contact symmetry algebra $\operatorname{Sym}_{0}(\mathrm{dVN})$ is generated by functions

$$
\begin{aligned}
\phi_{0}(A) & =-A u_{t}-\frac{1}{3} A^{\prime}\left(x u_{x}+y u_{y}\right)-\frac{1}{18} A^{\prime \prime}\left(x^{3}+y^{3}\right), \\
\phi_{1,1}(A) & =-A u_{x}-\frac{1}{2} A^{\prime} x^{2} \\
\phi_{2,1}(A) & =-A u_{y}-\frac{1}{2} A^{\prime} y^{2} \\
\phi_{1,2}(A) & =-A x \\
\phi_{2,2}(A) & =-A y \\
\phi_{3}(A) & =A \\
\psi & =3 u-x u_{x}-y u_{y}
\end{aligned}
$$

where $A=A(t)$ and $B=B(t)$ below are arbitrary smooth functions of $t$. Actually, all the contact symmetries of (1) turn out to be point symmetries. The structure of the Lie algebra $\operatorname{Sym}_{0}(\mathrm{dVN})$ is defined by the commutator table

$$
\begin{aligned}
\left\{\phi_{0}(A), \phi_{0}(B)\right\} & =\phi_{0}\left(A B^{\prime}-B A^{\prime}\right), \\
\left\{\phi_{0}(A) \phi_{i, j}(B)\right\} & =\phi_{i, j}\left(A B^{\prime}-\left(1-\frac{2}{3} j\right) B A^{\prime}\right), \quad i, j \in\{1,2\}, \\
\left\{\phi_{0}(A), \phi_{3}(B)\right\} & =\phi_{3}\left(A B^{\prime}\right), \\
\left\{\phi_{i, 1}(A), \phi_{j, 1}(B)\right\} & =\delta_{i j} \phi_{i, 2}\left(A B^{\prime}-B A^{\prime}\right),
\end{aligned}
$$

\footnotetext{
1 Computations of symmetries, their commutators, cosymmetries, and conservation laws were supported by the Jets software [1].
} 


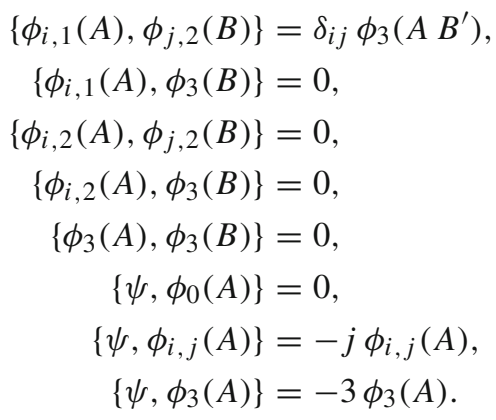

Direct computations show that the contact symmetry pseudogroup of equation (1) is generated by the infinite prolongations of the (local) diffeomorphisms $\Gamma_{0}:(t, x, y, u) \mapsto(\tilde{t}, \tilde{x}, \tilde{y}, \tilde{u})$ of the form

$$
\left\{\begin{aligned}
\tilde{t}= & B_{0}, \quad \tilde{x}=\varepsilon\left(B_{0}^{\prime} x+B_{1}\right), \quad \tilde{y}=\varepsilon\left(B_{0}^{\prime} y+B_{2}\right), \\
\tilde{u}= & \varepsilon^{3}\left(u-\frac{B_{0}^{\prime \prime}}{18 B_{0}^{\prime}}\left(x^{3}+y^{3}\right)-\frac{1}{2\left(B_{0}^{\prime}\right)^{1 / 3}}\left(B_{1}^{\prime} x^{2}+B_{2}^{\prime} y^{2}\right)\right. \\
& \left.+B_{3} x+B_{4} y+B_{5}\right),
\end{aligned}\right.
$$

where $\varepsilon \neq 0, B_{i}=B_{i}(t)$ are arbitrary functions, $B_{i}^{\prime}=\frac{d B_{i}}{d t}$, and $B_{0}^{\prime}(t) \neq 0$. In other words, substitution for (5) into dVN written in the tilded variables yields (1).

\subsection{The optimal system of one-dimensional subalgebras}

Since the symmetry algebra of dVN is infinite-dimensional and depends on 6 arbitrary functions of one variable, the problem of examining all invariant solutions is very complicated. To overcome the difficulty, we use the following observation: transformations from the symmetry pseudogroup (5) preserve equation (1), while changing the symmetry generators. Therefore we can classify the orbits of the action of (5) on the $\operatorname{Sym}_{0}(\mathrm{dVN})$. In order to use symmetries for computing invariant solutions we consider symmetries whose generators depend explicitly on at least one of the variables $u, u_{t}$, $u_{x}$, or $u_{y}$.

\section{Proposition 1 Each symmetry}

$\Phi=\phi_{0}\left(A_{0}\right)+\phi_{1,1}\left(A_{1,1}\right)+\phi_{2,1}\left(A_{2,1}\right)+\phi_{2,1}\left(A_{2,1}\right)+\phi_{2,2}\left(A_{2,2}\right)+\phi_{3}\left(A_{3}\right)+\mu \psi$

from $\operatorname{Sym}_{0}(\mathrm{dVN})$ with $A_{0}^{2}+\mu^{2}+A_{1,1}^{2}+A_{2,1}^{2} \not \equiv 0$ is equivalent with respect to the action of the pseudogroup (5) to one of symmetries

$$
\begin{aligned}
& \chi_{1}=\phi_{0}(1)+\mu \psi=-u_{t}+\mu\left(3 u-x u_{x}-y u_{y}\right), \\
& \chi_{2}=\psi=3 u-x u_{x}-y u_{y}, \\
& \chi_{3}=\phi_{1,1}(1)+\phi_{2,1}(A)=-u_{x}-A u_{y}-\frac{1}{2} A^{\prime} y^{2},
\end{aligned}
$$




$$
\chi_{4}=\phi_{2,1}(1)+\phi_{1,2}(A)=-u_{y}-A x .
$$

Proof Let $A_{0}(t) \neq 0$. Put $\varepsilon=1$ and consider solutions $B_{0}(t), \ldots, B_{5}(t)$ to the system of ODES

$$
\left\{\begin{array}{l}
B_{0}^{\prime}=A_{0}^{-1}, \\
B_{1}^{\prime}=\mu A_{0}^{-1} B_{1}-A_{0}^{-4 / 3} A_{1,1}, \\
B_{2}^{\prime}=\mu A_{0}^{-1} B_{2}-A_{0}^{-4 / 3} A_{2,1}, \\
B_{3}^{\prime}=\frac{1}{3} A_{0}^{-1}\left(6 \mu-A_{0}^{\prime}\right) B_{3}+\mu A_{0}^{-5 / 3} A_{1,1} B_{1}-A_{0}^{-2}\left(A_{0} A_{1,2}+A_{1,1}^{2}\right), \\
B_{4}^{\prime}=\frac{1}{3} A_{0}^{-1}\left(6 \mu-A_{0}^{\prime}\right) B_{4}+\mu A_{0}^{-5 / 3} A_{2,1} B_{2}-A_{0}^{-2}\left(A_{0} A_{2,2}+A_{2,1}^{2}\right), \\
B_{5}^{\prime}=A_{0}^{-1}\left(3 \mu B_{5}-A_{1,1} B_{3}-A_{2,1} B_{4}-A_{3}\right) .
\end{array}\right.
$$

Direct computations show that for $\tilde{\chi}_{1}=-\tilde{u}_{\tilde{t}}+\mu\left(3 \tilde{u}-\tilde{x} \tilde{u}_{\tilde{x}}-\tilde{y} \tilde{u}_{\tilde{y}}\right)$ there holds $\Gamma_{1}^{*}\left(\tilde{\chi}_{1}\right)=\Phi$, where $\Gamma_{1}$ is the first prolongation of (5). Therefore the inverse diffeomorphism maps $\Phi$ to $\tilde{\chi}_{1}$.

When $A_{0}(t) \equiv 0$ and $\mu \neq 0$, put $\varepsilon=\mu^{1 / 3}$ and define (5) by $B_{0}=t, B_{1}=$ $\mu^{-1} A_{1,1}, B_{2}=\mu^{-1} A_{2,1}, B_{3}=\frac{1}{2} \mu^{-2}\left(\mu A_{1,2}-A_{1,1} A_{1,1}^{\prime}\right), B_{4}=\frac{1}{2} \mu^{-2}\left(\mu A_{2,2}-\right.$ $\left.A_{2,1} A_{2,1}^{\prime}\right), B_{5}=\frac{1}{6} \mu^{-3}\left(2 \mu^{2} A_{3}+\mu\left(A_{1,1} A_{1,2}+A_{2,1} A_{2,2}\right)-A_{1,1}^{2} A_{1,1}^{\prime}-A_{2,1}^{2} A_{2,1}^{\prime}\right)$. Then we have $\Gamma_{1}^{*}\left(\tilde{\chi}_{2}\right)=\left.\Phi\right|_{A_{0}=0}$.

Suppose now that $A_{0}(t) \equiv 0, \mu=0, A_{1,1}(t) \neq 0$, and $A_{2,1}(t) \neq 0$. Put $\varepsilon=1$ and define functions $B_{i}, A(\tilde{t})$ by equations $B_{0}^{\prime}=A_{1,1}^{-3}, B_{1}^{\prime}=A_{1,2} A_{1,1}^{-2}, B_{2}^{\prime}=\left(A_{2,2}+\right.$ $\left.\left(A_{1,1} A_{2,1}^{\prime}-A_{2,1} A_{1,1}^{\prime}\right) B_{2}\right) A_{1,1}^{-1} A_{2,1}^{-1}, B_{3}=\frac{1}{2}\left(A_{1,1} A_{2,1}^{\prime}-A_{2,1} A_{1,1}^{\prime}\right) B_{2}^{2}-A_{3} A_{1,1}^{-1}$, $B_{4}=0$, and $A\left(B_{0}(t)\right)=A_{2,1}(t) / A_{1,1}(t)$. Then $\Gamma_{1}^{*}\left(\tilde{\chi}_{3}\right)=\left.\Phi\right|_{A_{0}=0, \mu=0}$.

Finally, when $A_{0}(t) \equiv 0, \mu=0, A_{1,1}(t) \equiv 0$, and $A_{2,1}(t) \neq 0$, put $\varepsilon=1$ and define functions $B_{i}, A(\tilde{t})$ by equations $B_{0}^{\prime}=A_{2,1}^{-3}, B_{1} \equiv 0, B_{2}=A_{2,2} A_{2,1}^{-2}, B_{3} \equiv 0, B_{4}=$ $-A_{3} A_{2,1}^{-1}$, and $A\left(B_{0}(t)\right)=A_{1,2}(t) A_{2,1}(t)$. Then $\Gamma_{1}^{*}\left(\tilde{\chi}_{4}\right)=\left.\Phi\right|_{A_{0}=0, \mu=0, A_{1,1}=0}$.

\section{Invariant solutions}

In this section we analyze reductions of $\mathrm{dVN}$ with respect to the symmetries $\chi_{1}, \ldots$, $\chi_{4}$.

\subsection{Reduction w.r.t. $\chi_{1}$}

The $\chi_{1}$-invariant solutions of dVN satisfy equation (1) and

$$
\chi_{1}=-u_{t}+\mu\left(3 u-x u_{x}-y u_{y}\right)=0,
$$

so they are of the form

$$
u=\mathrm{e}^{3 \mu t} \hat{U}(s, w), \quad s=x \mathrm{e}^{-\mu t}, \quad w=y \mathrm{e}^{-\mu t} .
$$


Table 1 Commutator table of the Lie algebra $\operatorname{Sym}_{0}\left(\varepsilon_{\mu}\right)$

\begin{tabular}{llllll}
\hline & $\eta_{2}$ & $\eta_{3}$ & $\eta_{4}$ & $\eta_{5}$ & $\eta_{6}$ \\
\hline$\eta_{1}$ & $-\eta_{2}$ & $-\eta_{3}$ & $-2 \eta_{4}$ & $-2 \eta_{5}$ & $-3 \eta_{6}$ \\
$\eta_{2}$ & & 0 & $\eta_{6}$ & 0 & 0 \\
$\eta_{3}$ & & & 0 & $\eta_{6}$ & 0 \\
$\eta_{4}$ & & & & 0 & 0 \\
$\eta_{5}$ & & & & & 0 \\
\hline
\end{tabular}

We introduce function

$$
\hat{U}=U-\frac{\mu}{6}\left(s^{3}+w^{3}\right)
$$

for convenience of the further computations. Substitution for (6), (7) into (1) shows that $U(z, w)$ is a solution to equation $\mathcal{E}_{\mu}$ defined by

$$
U_{w w w}=-U_{s s s}-\frac{U_{s s} U_{s s w}+U_{w w} U_{s w w}}{U_{s w}}+3 \mu .
$$

This equation admits a Lax representation. Indeed, symmetry $\chi_{1}$ has the lift $\left(\chi_{1}, \hat{\chi}_{1}\right)$ with $\hat{\chi}_{1}=-q_{t}+\mu\left(\frac{3}{2} q-x q_{x}-y q_{y}\right)$ to the Lax representation (2). Solutions to equation $\hat{\chi}_{1}=0$ are of the form $q=\mathrm{e}^{\frac{3}{2} \mu t} Q(s, w)$. Substituting this into (2) yields the Lax representation

$$
\left\{\begin{array}{l}
Q_{s}^{6}=-3 U_{s s} Q_{s}^{4}+\frac{9}{2} \mu Q Q_{s}^{3}+3 U_{s w} U_{w w} Q_{s}^{2}+U_{s w}^{3} \\
Q_{w}=-\frac{U_{s w}}{Q_{s}}
\end{array}\right.
$$

for equation (8). We put $Q_{s}=S$ and obtain another Lax representation for (8):

$$
\left\{\begin{array}{l}
S_{s}=-\frac{S}{2} \frac{\left(3 \mu+2 U_{s s s}\right) S^{4}-2\left(U_{w w} U_{s s w}+U_{s w} U_{s w w}\right) S^{2}-2 U_{s w}^{2} U_{s s w}}{S^{6}+U_{s s} S^{4}+U_{s w} U_{w w} S^{2}+U_{s w}^{3}} \\
S_{w}=-\frac{S}{2} \frac{2 U_{s s w} S^{4}+\left(2 U_{s s} U_{s s w}+U_{s w}\left(2 U_{s s s}+3 \mu\right) S^{2}-2 U_{s w} U_{s w w}\right.}{S^{6}+U_{s s} S^{4}+U_{s w} U_{w w} S^{2}+U_{s w}^{3}}
\end{array}\right.
$$

Now we analyze invariant solutions to equation (8). We consider cases $\mu \neq 0$ and $\mu=0$ separately.

\subsubsection{Case $\mu \neq 0$.}

When $\mu \neq 0$, the symmetry algebra $\operatorname{Sym}_{0}\left(\varepsilon_{\mu}\right)$ of equation (8) is generated by functions $\eta_{1}=3 U-s U_{s}-w U_{w}, \eta_{2}=-U_{s}, \eta_{3}=-U_{w}, \eta_{4}=s, \eta_{5}=w, \eta_{6}=1$. The structure of this Lie algebra is defined by the commutators in Table 1. 
Table 2 The adjoint reperesentation of the Lie group $G_{\mu}$

\begin{tabular}{lllllll}
\hline & $\eta_{1}$ & $\eta_{2}$ & $\eta_{3}$ & $\eta_{4}$ & $\eta_{5}$ & $\eta_{6}$ \\
\hline$\eta_{1}$ & $\eta_{1}$ & $\mathrm{e}^{-\tau} \eta_{2}$ & $\mathrm{e}^{-\tau} \eta_{3}$ & $\mathrm{e}^{-2 \tau} \eta_{4}$ & $\mathrm{e}^{-2 \tau} \eta_{5}$ & $\mathrm{e}^{-3 \tau} \eta_{6}$ \\
$\eta_{2}$ & $\eta_{1}+\tau \eta_{2}$ & $\eta_{2}$ & $\eta_{3}$ & $\eta_{4}+\tau \eta_{6}$ & $\eta_{5}$ & $\eta_{6}$ \\
$\eta_{3}$ & $\eta_{1}+\tau \eta_{3}$ & $\eta_{2}$ & $\eta_{3}$ & $\eta_{4}$ & $\eta_{5}+\tau \eta_{6}$ & $\eta_{6}$ \\
$\eta_{4}$ & $\eta_{1}+2 \tau \eta_{4}$ & $\eta_{2}-\tau \eta_{6}$ & $\eta_{3}$ & $\eta_{4}$ & $\eta_{5}$ & $\eta_{6}$ \\
$\eta_{5}$ & $\eta_{1}+2 \tau \eta_{5}$ & $\eta_{2}$ & $\eta_{3}-\tau \eta_{6}$ & $\eta_{4}$ & $\eta_{5}$ & $\eta_{6}$ \\
$\eta_{6}$ & $\eta_{1}+3 \tau \eta_{6}$ & $\eta_{2}$ & $\eta_{3}$ & $\eta_{4}$ & $\eta_{5}$ & $\eta_{6}$ \\
\hline
\end{tabular}

The adjoint representation of the Lie group $G_{\mu}$ associated with the finitedimensional Lie algebra $\operatorname{Sym}_{0}\left(\mathcal{E}_{\mu}\right)$ is defined by the Lie series

$$
\operatorname{Ad}_{\tau \eta_{i}}\left(\eta_{j}\right)=\exp \left(\tau \operatorname{ad} \eta_{i}\right)\left(\eta_{j}\right)=\sum_{k \geq 0} \frac{\tau^{k}}{k !}\left(\operatorname{ad} \eta_{i}\right)^{k}\left(\eta_{j}\right),
$$

where ad $\eta_{i}\left(\eta_{j}\right)=\left\{\eta_{i}, \eta_{j}\right\}$. This representation is given by Table 2 . In this table the $(i, j)$-th entry is the expression for $\operatorname{Ad}_{\tau \eta_{i}}\left(\eta_{j}\right)$. Using this table one can classify the orbits of action of the adjoint representation of the Lie group $G_{\mu}$ on its Lie algebra $\operatorname{Sym}_{0}\left(\varepsilon_{\mu}\right)$ :

Proposition 2 Each symmetry of equation $\mathcal{E}_{\mu}$ with $\mu \neq 0$ is equivalent under the action of the adjoint representation of $G_{\mu}$ to one of the symmetries

$$
\begin{aligned}
& \zeta_{1}=\eta_{1}=3 U-s U_{s}-w U_{w}, \\
& \zeta_{2}=\eta_{3}+\alpha \eta_{2}+\beta \eta_{4}+\gamma \eta_{5}=-U_{w}-\alpha U_{s}+\beta s+\gamma w, \\
& \zeta_{3}=\eta_{2}+\beta \eta_{4}+\gamma \eta_{5}=-U_{s}+\beta s+\gamma w,
\end{aligned}
$$

where $\alpha, \beta, \gamma \in \mathbb{R}$.

Proof is obtained by the standard computation, see, e.g., [18, § 3.3].

Thus each invariant solution to equation (8) can be obtained by an action of appropriate superposition of transformations $\operatorname{Ad}_{\tau} \eta_{i}\left(\eta_{j}\right)$ from $\zeta_{k}$-invariant solutions. Below we examine such solutions.

\subsubsection{Solutions invariant w.r.t. $\zeta_{1}$.}

The $\zeta_{1}$-invariant solutions satisfy $(8)$ and $\zeta_{1}=0$, therefore they are of the form

$$
U=s^{3} W(z), \quad z=w s^{-1},
$$

where $W$ is a solution to the ODE

$$
W_{z z z}=\frac{\left(5 z^{3}-1\right) W_{z z}^{2}-z\left(22 z W_{z}-18 W+3 \mu\right) W_{z z}}{2\left(\left(z^{3}-1\right) W_{z z}-\left(3 z^{3}-1\right) W_{z}+3 z^{2} W\right)}
$$




$$
+\frac{20 z W_{z}^{2}-6(4 W-\mu) W_{z}}{2\left(\left(z^{3}-1\right) W_{z z}-\left(3 z^{3}-1\right) W_{z}+3 z^{2} W\right)} .
$$

We could not obtain the general solution to this ODE. Instead, we find a family of particular solutions given by

$$
W=\frac{1}{18 a_{1} a_{2}}\left(\left(3 \mu a_{1} a_{2}-4 a_{1}^{3}+2 a_{2}^{3}\right) z^{3}+3 \mu a_{1} a_{2}-4 a_{2}^{3}+2 a_{1}^{3}\right)+a_{2} z^{2}+a_{1} z
$$

where $a_{1} a_{2} \neq 0$. Then (6), (7), (9) give the $t$-independent solution

$$
u=x y\left(a_{1} x+a_{2} y\right)+\frac{1}{9 a_{1} a_{2}}\left(\left(a_{1}^{3}-2 a_{2}^{3}\right) x^{3}+\left(a_{2}^{3}-2 a_{1}^{3}\right) y^{3}\right)
$$

to dVN.

\subsubsection{Solutions invariant w.r.t. $\zeta_{2}$.}

For $\zeta_{2}$-invariant solutions there holds $\zeta_{2}=0$, or

$$
U_{w}+\alpha U_{s}-\beta s-\gamma w=0 .
$$

When $\alpha \neq 0$, this equation gives

$$
U=V(z)+\frac{\beta}{2 \alpha} s^{2}+\frac{\gamma}{2} w^{2}, \quad z=s-\alpha w
$$

Substituting for (12) into (8) yields

$$
V_{z z z}=-\frac{3 \alpha \mu V_{z z}}{2 \alpha\left(\alpha^{3}-1\right) V_{z z}-\beta+\alpha^{2} \gamma}
$$

This equation is integrable by quadratures. When $\beta=\alpha^{2} \gamma, \alpha \neq-1$, the general solution to (13) is

$$
V=-\frac{\mu}{4\left(\alpha^{3}-1\right)} z^{3}+c_{2} z^{2}+c_{1} z+c_{0}
$$

where $c_{0}, c_{1}, c_{2}$ are arbitrary constants. This function produces solution

$$
\begin{gathered}
u=\frac{\mu}{12\left(\alpha^{3}-1\right)}\left(\left(\alpha^{3}+2\right) y^{3}-\left(2 \alpha^{3}+1\right) x^{3}\right)+\frac{3 \alpha \mu}{4\left(\alpha^{3}-1\right)} x y(x-\alpha y) \\
+\frac{1}{2} \mathrm{e}^{\mu t}\left(\left(\alpha \gamma+2 c_{2}\right) x^{2}+\left(\gamma+2 c_{2} \alpha^{2}\right) y^{2}-4 c_{2} \alpha x y\right) \\
+c_{1} \mathrm{e}^{2 \mu t}(x-\alpha y)+c_{0} \mathrm{e}^{3 \mu t}
\end{gathered}
$$

of dVN. 
Remark 1 If $u$ is a solution to (1), then for arbitrary functions $b_{0}(t), b_{1}(t), b_{2}(t)$ the linear combination $u+b_{0}(t)+b_{1}(t) x+b_{2}(t) y$ is a solution to dVN as well. So we can put $c_{0}=c_{1}=0$ in (14) without loss of generality. In what follows we will ignore the linear in $x$ and $y$ terms in solutions of $\mathrm{dVN}$.

When $\beta \neq \alpha^{2} \gamma, \alpha \neq-1$, equation (13) has the first integral

$$
2 \alpha\left(\alpha^{3}-1\right) V_{z z}+\left(\alpha^{2} \gamma-\beta\right) \ln V_{z z}=-3 \alpha \mu z+c_{0}, \quad c_{0} \in \mathbb{R} .
$$

hence the general solution in this case is of form

$$
V=\int\left(\int H\left(-3 \alpha \mu z+c_{0}\right) d z\right) d z
$$

where function $H(\tau)$ is defined by formula

$$
2 \alpha\left(\alpha^{3}-1\right) H(\tau)+\left(\alpha^{2} \gamma-\beta\right) \ln H(\tau) \equiv \tau
$$

This function can be expressed in terms of the Lambert $\mathrm{W}$ function, [6], while the expression is too complicated to be written here.

When $\alpha=-1, \beta \neq \gamma$, the general solution of (13) reads

$$
V=c_{2} \exp \left(\frac{3 \mu z}{\beta-\gamma}\right)+c_{1} z+c_{0}, \quad c_{i} \in \mathbb{R}
$$

to dVN of the form

$$
u=c_{2} \exp \left(3 \mu\left(t+\mathrm{e}^{-\mu t} \frac{x-y}{\beta-\gamma}\right)\right)-\frac{\mu}{6}\left(x^{3}+y^{3}\right)+\frac{1}{2} \mathrm{e}^{\mu t}\left(\beta x^{2}+\gamma y^{2}\right),
$$

cf. Remark 1.

Finally, when $\alpha=0$ we have solution

$$
U=\frac{\mu}{2} s^{3}+\frac{\gamma}{2} w^{2}+\beta s w+c_{2} z^{2}+c_{1} z+c_{0}
$$

of equation (8). This produces the following solution to $\mathrm{dVN}$ :

$$
u=\frac{\mu}{6}\left(2 x^{3}-y^{3}\right)+\mathrm{e}^{\mu t}\left(\beta x y+c_{2} x^{2}+\gamma y^{2}\right) .
$$

\subsubsection{Solutions invariant w.r.t. $\zeta_{3}$}

The $\zeta_{3}$-invariant solution of equation (8) has the form

$$
U=\frac{\beta}{2} s^{2}+\gamma s w+c_{0}+c_{1} w+c_{2} w^{3}+\frac{\mu}{2} w^{3}, \quad c_{i} \in \mathbb{R},
$$

the corresponding solution of $\mathrm{dVN}$ is obtained from (18) by renaming $x \leftrightarrow y, \beta \leftrightarrow \gamma$. 
Table 3 Commutator table of the Lie algebra $\operatorname{Sym}_{0}\left(\varepsilon_{0}\right)$

\begin{tabular}{|c|c|c|c|c|c|c|}
\hline & $\xi_{2}$ & $\xi_{3}$ & $\xi_{4}$ & $\xi_{5}$ & $\xi_{6}$ & $\xi_{7}$ \\
\hline$\xi_{1}$ & $-\xi_{2}$ & $-\xi_{3}$ & $\xi_{4}$ & $\xi_{5}$ & 0 & 0 \\
\hline$\xi_{2}$ & & 0 & $\xi_{6}$ & 0 & 0 & 0 \\
\hline$\xi_{3}$ & & & 0 & $\xi_{6}$ & 0 & 0 \\
\hline$\xi_{4}$ & & & & 0 & 0 & $\xi_{4}$ \\
\hline$\xi_{5}$ & & & & & 0 & $\xi_{5}$ \\
\hline$\xi_{6}$ & & & & & & $\xi_{6}$ \\
\hline
\end{tabular}

Table 4 The adjoint action of the Lie group $G_{0}$

\begin{tabular}{llllllll}
\hline & $\xi_{1}$ & $\xi_{2}$ & $\xi_{3}$ & $\xi_{4}$ & $\xi_{5}$ & $\xi_{6}$ & $\xi_{7}$ \\
\hline$\xi_{1}$ & $\xi_{1}$ & $\mathrm{e}^{-\tau} \xi_{2}$ & $\mathrm{e}^{-\tau} \xi_{3}$ & $\mathrm{e}^{\tau} \xi_{4}$ & $\mathrm{e}^{\tau} \xi_{5}$ & $\xi_{6}$ & $\xi_{7}$ \\
$\xi_{2}$ & $\xi_{1}+\tau \xi_{2}$ & $\xi_{2}$ & $\xi_{3}$ & $\xi_{4}+\tau \xi_{6}$ & $\xi_{5}$ & $\xi_{6}$ & $\xi_{7}$ \\
$\xi_{3}$ & $\xi_{1}+\tau \xi_{3}$ & $\xi_{2}$ & $\xi_{3}$ & $\xi_{4}$ & $\xi_{5}+\tau \xi_{6}$ & $\xi_{6}$ & $\xi_{7}$ \\
$\xi_{4}$ & $\xi_{1}-\tau \xi_{4}$ & $\xi_{2}-\tau \xi_{6}$ & $\xi_{3}$ & $\xi_{4}$ & $\xi_{5}$ & $\xi_{6}$ & $\xi_{7}-\tau \xi_{4}$ \\
$\xi_{5}$ & $\xi_{1}-\tau \xi_{5}$ & $\xi_{2}$ & $\xi_{3}-\tau \xi_{6}$ & $\xi_{4}$ & $\xi_{5}$ & $\xi_{6}$ & $\xi_{7}-\tau \xi_{5}$ \\
$\xi_{6}$ & $\xi_{1}$ & $\xi_{2}$ & $\xi_{3}$ & $\xi_{4}$ & $\xi_{5}$ & $\xi_{6}$ & $\xi_{7}-\tau \xi_{6}$ \\
$\xi_{6}$ & $\xi_{1}$ & $\xi_{2}$ & $\xi_{3}$ & $\mathrm{e}^{-\tau} \xi_{4}$ & $\mathrm{e}^{-\tau} \xi_{5}$ & $\mathrm{e}^{-\tau} \xi_{6}$ & $\xi_{7}$ \\
\hline
\end{tabular}

Remark 2 For each solution $u=f(t, x, y)$ of $\mathrm{dVN}$ expression $u=f(t, y, x)$ defines a solution as well.

\subsubsection{Case $\mu=0$}

Now consider equation $\mathcal{E}_{0}$ obtained by putting $\mu=0$ in (8). Notice that solutions of this equation produce $t$-independent solutions to $\mathrm{dVN}$, and $t$-independent solutions are defined up to a nonzero constant multiple. The symmetry algebra $\operatorname{Sym}_{0}\left(\mathcal{E}_{0}\right)$ of this equation has generators $\xi_{1}=-s U_{s}-w U_{w}, \xi_{2}=-U_{s}, \xi_{3}=-U_{w}, \xi_{4}=s$, $\xi_{5}=w, \xi_{6}=1$, and $\xi_{7}=U$, with the commutators given in Table 3 .

The adjoint action of the symmetry group $G_{0}$ of equation $\mathcal{E}_{0}$ on $\operatorname{Sym}_{0}\left(\mathcal{E}_{0}\right)$ is defined by the Table 4 . Then direct computations give the optimal system of one-dimensional subalgebras of $\operatorname{Sym}_{0}\left(\mathcal{E}_{0}\right)$ :

Proposition 3 Each symmetry of equation $\mathcal{E}_{0}$ is equivalent under the action of the adjoint representation of $G_{0}$ to one of the following symmetries:

$$
\begin{aligned}
& \sigma_{1}=\xi_{1}+\alpha \xi_{7}=-s U_{s}-w U_{w}+\alpha U, \\
& \sigma_{2}=\xi_{1}+\alpha \xi_{6}=-s U_{s}-w U_{w}+\alpha, \\
& \sigma_{3}=\xi_{1}+\xi_{7}+\xi_{4}+\alpha \xi_{5}=-s U_{s}-w U_{w}+U+s+\alpha w, \\
& \sigma_{4}=\xi_{1}+\xi_{7}+\xi_{5}=-s U_{s}-w U_{w}+U+w, \\
& \sigma_{5}=\xi_{2}+\alpha \xi_{3}+\beta \xi_{7}=-U_{s}-\alpha U_{w}+\beta U, \quad \alpha \neq 0 \\
& \sigma_{6}=\xi_{2}+\alpha \xi_{3}+\xi_{4}+\beta \xi_{5}=-U_{s}-\alpha U_{w}+s+\beta w,
\end{aligned}
$$




$$
\begin{aligned}
& \sigma_{7}=\xi_{2}+\alpha \xi_{3}+\xi_{5}=-U_{s}-\alpha U_{w}+w, \\
& \sigma_{8}=\xi_{2}+\alpha \xi_{3}=-U_{s}-\alpha U_{w}, \\
& \sigma_{9}=\xi_{3}+\alpha \xi_{7}=-U_{w}+\alpha U, \\
& \sigma_{10}=\xi_{3}+\alpha \xi_{4}+\beta \xi_{5}=-U_{w}+\alpha s+\beta w .
\end{aligned}
$$

\subsubsection{Solutions invariant w.r.t. $\sigma_{1}$.}

The $\sigma_{1}$-invariant solutions to $\varepsilon_{0}$ have the form

$$
U=s^{\alpha} W(z), \quad z=w s^{-1} .
$$

Substituting this into (8) gives the ODE

$$
W_{z z z}=(\alpha-2) \frac{F\left(z, W, W_{z}, W_{z z}\right)}{G\left(z, W, W_{z}, W_{z z}\right)} \text {, }
$$

where

$$
\begin{aligned}
& F\left(z, W, W_{z}, W_{z z}\right)=\left(5 z^{3}-1\right) W_{z z}^{2}-(\alpha-1) z\left(11 z W_{z}-3 \alpha W\right) W_{z z} \\
& \quad+(\alpha-1)^{2}\left(5 z W_{z}-2 \alpha W\right) W_{z}
\end{aligned}
$$

and

$$
G\left(z, W, W_{z}, W_{z z}\right)=2 z\left(z^{3}-1\right) W_{z z}-(\alpha-1)\left(3 z^{3}-1\right) W_{z}+\alpha(\alpha-1) z^{2} W .
$$

We did not find the general solution to this equation for any $\alpha$. For each $\alpha$ equation (19) admits solutions of the form

$$
W=\left(z+z_{0}\right)^{\alpha},
$$

where $z_{0}$ is a root of equation $z_{0}\left(z_{0}^{3}+1\right)=0$. Thus we get solution

$$
u=\left(y+z_{0} x\right)^{\alpha}
$$

of equation (1). We found some other solutions of (19) for $\alpha \in\{1,2,3,4\}$.

Equation (19) with $\alpha=1$

$$
W_{z z z}=-\frac{1}{2} \frac{5 z^{3}-1}{z\left(z^{3}-1\right)} W_{z z} .
$$

is integrable by quadratures, its general solution

$$
W(z)=c_{0}+c_{1} z+\int\left(\int z^{-1 / 2}\left(z^{3}-1\right)^{-2 / 3} d z\right) d z
$$


produces solution to $\mathrm{dVN}$ of the form

$$
u=x^{4} W\left(y x^{-1}\right)
$$

Notice that integral in (22) can not be expressed in elementary functions, [3, § VIII], [22, Ch. 3, § 14].

When $\alpha=2$, equation (19) reduces to $W_{z z z}=0$, its general solution $W=$ $c_{0}+c_{1} z+c_{2} z^{2}, c_{i} \in \mathbb{R}$, gives solution of dVN

$$
u=c_{0} x^{2}+c_{1} x y+c_{2} y^{2} .
$$

Remark 3 Expression $u=b_{1}(t) x^{2}+c x y+b_{2}(t) y^{2}$ with arbitrary functions $b_{1}(t)$, $b_{2}(t)$ and constant $c$ defines a trivial solution to dVN. We will ignore such solutions below.

For $\alpha=3$ we obtain two algebraic solutions

$$
W=z^{\frac{3}{2}}
$$

and

$$
W=\left(c_{2}^{3}-2 c_{1}^{3}\right) z^{3}+9 c_{1} c_{2} z\left(c_{2} z+c_{1}\right)+c_{1}^{3}-2 c_{2}^{3}
$$

of (19). They give solutions

$$
u=x^{\frac{3}{2}} y^{\frac{3}{2}}
$$

and

$$
u=\left(c_{2}^{3}-2 c_{1}^{3}\right) y^{3}+9 c_{1} c_{2}^{2} x y\left(c_{2} y+c_{1} x\right)+\left(c_{1}^{3}-2 c_{2}^{3}\right) x^{3}
$$

of dVN.

When $\alpha=4$, equation (19) admits the polynomial solution $W=17 z^{4}-36 z^{3}-$ $90 z^{2}-36 z+17$. This corresponds to solution of $\mathrm{dVN}$ of the form

$$
u=17 x^{4}-36 x^{3} y-90 x^{2} y^{2}-36 x y^{3}+17 y^{4} .
$$

\subsubsection{Solutions invariant w.r.t. $\sigma_{2}$.}

For $\sigma_{2}$-invariant solutions of $\mathcal{E}_{0}$ there holds $\sigma_{2}=-U_{w}+\alpha U=0$, therefore these solutions have the form

$$
U=\mathrm{e}^{\alpha w} V(s)
$$

Substituting this into $\varepsilon_{0}$ gives the reduced equation $V_{s} V_{s s s}+V_{s s}^{2}+2 \alpha^{3} V V_{s}=0$. Integrating this twice, we obtain the first order ODE

$$
V_{s}^{3}+\alpha^{3}\left(V^{3}+\gamma V+\delta\right)=0
$$


with arbitrary constants $\gamma$ and $\delta$. This equation is integrable by quadratures, and the general solution can be expressed in elliptic functions, [10, Ch. V, $\S 21]$. To prove this claim, consider transformation

$$
\left\{\begin{array}{l}
P=\frac{\gamma^{1 / 2}\left(3 p^{2}+r-1\right)}{6 p}, \\
R=-\frac{\gamma^{1 / 2}\left(3 p^{2}-r+1\right)}{6 p},
\end{array}\right.
$$

that maps the curve $R^{3}=P^{3}+\gamma P+\delta$ onto the curve

$$
r^{2}=1-2 \beta p-6 p^{2}-3 p^{4}
$$

with $\beta=6 \delta \gamma^{-3 / 2}$. We have $2 r d r=-2\left(\beta+6 p+6 p^{3}\right) d p$, hence

$$
\frac{d P}{R}=\frac{1}{2 p}\left(\frac{3 p^{2}+1}{r}-1\right) d p .
$$

Then (29), (6), (7), and $\mu=0$ give solution

$$
u=\mathrm{e}^{\alpha y} V(x),
$$

where function $V$ is defined implicitly by equations

$$
\begin{aligned}
& V=\frac{\sqrt{\gamma}}{6 p}\left(3 p^{2}-1+\sqrt{1-2 \beta p-6 p^{2}-3 p^{4}}\right), \\
& \int \frac{1}{2 p}\left(1-\frac{3 p^{2}+1}{\sqrt{1-2 \beta p-6 p^{2}-3 p^{4}}}\right) d p=\alpha^{3} x+C .
\end{aligned}
$$

The integral in (34) includes Legendre's integrals, therefore $x$ can be expressed as an elliptic function of $p$.

When polynomial $V^{3}+\gamma V+\delta$ has multiple roots, the general solution to equation (30) can be expressed in elementary functions. Indeed, the presence of the triple root implies $\gamma=\delta=0$, and then solution to (30) is $V=c \mathrm{e}^{-\alpha s}, c \in \mathbb{R}$. This corresponds to solution

$$
u=\mathrm{e}^{\alpha(y-x)}
$$

of dVN. For a double root we have $V^{3}+\gamma V+\delta=(V+2 \varepsilon)(V-\varepsilon)^{2}$ for some $\varepsilon \neq 0$. Then we obtain solution to dVN of the form (32), where function $V$ is defined in the implicit form by equations

$$
V=\frac{\varepsilon\left(\tau^{3}+2\right)}{t^{3}-1},
$$




$$
\frac{1}{2} \ln \frac{\tau^{2}+\tau+1}{\tau-1}+\sqrt{3} \arctan \left(\frac{\sqrt{3}}{3}(2 \tau+1)\right)=-\alpha^{3} x+c
$$

with $c \in \mathbb{R}$.

There are some other values of parameters $\gamma$ and $\delta$ when integral $\int\left(V^{3}+\gamma V+\right.$ $\delta)^{-\frac{1}{3}} d V$ can be expressed by elementary functions. From the results of [4] (see also [9]) we obtain three following cases when function $V(x)$ in (32) admits elementary expressions in implicit form:

(i) for $\gamma=0, \delta \neq 0$ there holds

$$
\frac{3+i \sqrt{3}}{6}\left(\ln \left(V-\left(V^{3}+\delta\right)^{\frac{1}{3}}\right)+\mathrm{e}^{\frac{\pi i}{3}} \ln \left(V-\mathrm{e}^{\frac{2 \pi i}{3}}\left(V^{3}+\delta\right)^{\frac{1}{3}}\right)\right)=\alpha^{3} x+c,
$$

(ii) when $\gamma \neq 0, \delta=0$, we get

$$
\frac{3+i \sqrt{3}}{4}\left(\ln \left(V^{\frac{2}{3}}-\left(V^{2}+\gamma\right)^{\frac{1}{3}}\right)+\mathrm{e}^{\frac{\pi i}{3}} \ln \left(V^{\frac{2}{3}}-\mathrm{e}^{\frac{2 \pi i}{3}}\left(V^{2}+\gamma\right)^{\frac{1}{3}}\right)\right)=\alpha^{3} x+c
$$

(iii) conditions $\gamma^{3}=-6 \delta^{2} \neq 0$ imply

$$
\ln H(V, 1)+\mathrm{e}^{\frac{2 \pi i}{3}} \ln H\left(V, \mathrm{e}^{\frac{2 \pi i}{3}}\right)+\mathrm{e}^{\frac{4 \pi i}{3}} \ln H\left(V, \mathrm{e}^{\frac{4 \pi i}{3}}\right)=6 \alpha^{3} x+c,
$$

where

$$
H(\tau, \omega)=\frac{3 \delta \tau-\gamma(\tau+\gamma)-\omega(\gamma \tau+3 \delta) T+2 \gamma \omega^{2} T^{2}}{2 \gamma} \cdot(\tau-\omega T)
$$

with $T=\left(\tau^{2}+\gamma \tau+\delta\right)^{\frac{1}{3}}$.

4.1.2.3. Solutions invariant w.r.t. $\sigma_{3}, \sigma_{4}, \sigma_{5}$, and $\sigma_{6}$.

For symmetries $\sigma_{3}, \sigma_{4}, \sigma_{5}$, and $\sigma_{6}$ the reduced ODEs are of the form $W_{z z z}=0$. This gives only trivial solutions to dVN, cf. Remark 3 .

4.1.2.4. Solutions invariant w.r.t. $\sigma_{7}, \sigma_{8}, \sigma_{9}$, and $\sigma_{10}$.

For symmetries $\sigma_{7}, \ldots, \sigma_{10}$ we found neither general nor particular solutions of the reduced equations. Below we write the forms of the invariant solutions of $\mathrm{dVN}$ that include functions $W$ and reduced ODEs that define these functions.

For $\sigma_{7}$ we have

$$
u=\alpha \ln x+W\left(y x^{-1}\right)
$$

where $W(z)$ satisfies

$$
W_{z z z}=-2 \frac{\left(5 z^{3}-1\right) W_{z z}^{2}+z\left(11 z W_{z}-3 \alpha\right) W_{z z}+\left(5 z W_{z}-2 \alpha\right) W_{z}}{2 z\left(z^{3}-1\right) W_{z z}+\left(3 z^{3}-1\right) W_{z}-\alpha z^{2}} .
$$


The $\sigma_{8}$-invariant solutions are

$$
u=(x+\alpha y) \ln x+x W\left(y x^{-1}\right),
$$

where for $W(z)$ there holds

$$
W_{z z z}=-\frac{\left(5 z^{3}-1\right) W_{z z}^{2}-z(8 z-3 \alpha) W_{z z}+3 z-2 \alpha}{2 z\left(z^{3}-1\right) W_{z z}-2 z^{3}+\alpha z^{2}+1} .
$$

Symmetry $\sigma_{9}$ produces the invariant solution

$$
u=y\left(\ln y+W\left(y x^{-1}\right)\right)
$$

with the reduced ODE

$$
W_{z z z}=-\frac{z^{2}\left(11 z^{3}-7\right) W_{z z}^{2}+\left(16 z\left(2 z^{3}-1\right) W_{z}-3\right) W_{z z}+4\left(5 z^{3}-1\right) W_{z}}{2 z^{3}\left(z^{3}-1\right) W_{z z}+4 z^{2}\left(z^{3}-1\right) W_{z}-z}
$$

Finally, for $\sigma_{10}$ we get

$$
u=\mathrm{e}^{\beta x} W(y-\alpha x) .
$$

with the defining equation for $W(z)$ of the form

$$
\begin{aligned}
W_{z z z}= & \beta \frac{\left(5 \alpha^{3}-1\right) W_{z z}^{2}-\alpha \beta\left(11 \alpha W_{z}-3 \beta W\right) W_{z z}}{2 \alpha\left(\alpha^{3}-1\right) W_{z z}-\beta\left(3 \alpha^{3}-1\right) W_{z}+\alpha^{2} \beta^{2} W} \\
& +\beta^{3} \frac{5 \alpha W_{z}^{2}-4 \beta W W_{z}}{2 \alpha\left(\alpha^{3}-1\right) W_{z z}-\beta\left(3 \alpha^{3}-1\right) W_{z}+\alpha^{2} \beta^{2} W}
\end{aligned}
$$

\subsection{Reduction of dVN w.r.t. $\chi_{2}$}

The $\chi_{2}$-invariant solutions of dVN satisfy $\chi_{2}=3 u-x u_{x}-y u_{y}=0$, therefore they have the form $u=x^{3} U(t, z), z=y x^{-1}$, where $U$ is a solution to

$$
\begin{aligned}
& U_{t z z}=\frac{1}{z}\left(2 U_{t z}-2\left(z\left(z^{3}-1\right) U_{z z}-\left(3 z^{3}-1\right) U_{z}+3 z^{2} U\right) U_{z z z}\right. \\
& \left.\quad+\left(5 z^{3}-1\right) U_{z z}^{2}-2 z\left(11 z U_{z}-9 U\right) U_{z z}+4 U_{z}\left(5 z U_{z}-6 U\right)\right)
\end{aligned}
$$

This equation admits a Lax representation. To show this, consider the lift $\left(\chi_{2}, \hat{\chi}_{2}\right)$ of $\chi_{2}$ to the Lax representation (2) with $\hat{\chi}_{2}=\frac{3}{2} q-x q_{x}-y q_{y}$. Solutions to $\hat{\chi}_{2}=0$ 
have the form $q=x^{\frac{3}{2}} Q(t, z)$. Substituting this into (2) yields

$$
\left\{\begin{aligned}
Q_{t}= & \frac{2}{3}\left(z^{3}-1\right) Q_{z}^{3}-\frac{3\left(z^{3}-1\right)}{2 z} Q Q_{z}^{2}+\frac{2}{z}\left(\left(z^{3}+1\right) U_{z}-3 z^{2} U\right) Q_{z} \\
& +\frac{9}{8} Q^{3}-3\left(3 z U_{z}-3 U\right) Q \\
Q_{z}^{2}= & \frac{1}{2 z}\left(3 Q Q_{z}-2\left(z U_{z z}-2 U_{z}\right)\right) .
\end{aligned}\right.
$$

For $S=Q_{z}$ we obtain another Lax representation

$$
\left\{\begin{array}{l}
S_{t}=\frac{S^{6}+3 U_{z z} S^{4}+F\left(z, U, U_{z}, U_{z z}, U_{z z z}\right) S^{2}+G\left(z, U, U_{z}, U_{z z}\right)}{S\left(z S^{2}+2 U_{z}-U_{z z}\right)} \\
S_{z}=\frac{S\left(S^{2}+2 U_{z z}-2 z U_{z z z}\right)}{2\left(z S^{2}+2 U_{z}-U_{z z}\right)}
\end{array}\right.
$$

where

$$
\begin{aligned}
& F\left(z, U, U_{z}, U_{z z}, U_{z z z}\right)=4\left(z\left(z^{3}-1\right) U_{z z}-\left(3 z^{3}-1\right) U_{z}+3 z^{2} U\right) U_{z z z} \\
& \quad-\left(7 z^{2}-2\right) U_{z z}^{2}+2 z\left(13 U_{z}-9 U\right) U_{z z}-16 z U_{z}^{2}+12 U U_{z}, \\
& \quad G\left(z, U, U_{z}, U_{z z}\right)=z^{3} U_{z z}^{3}-6 z U_{z} U_{z z}\left(z U_{z z}-2 U_{z}\right)-8 U_{z}^{3} .
\end{aligned}
$$

The symmetry algebra of equation (50) is generated by the family $A(t) U_{t}+A^{\prime}(t) U+$ $\frac{1}{18} A^{\prime \prime}(t)\left(z^{3}+1\right)$ with arbitrary function $A(t)$. This symmetry provides invariant solutions to (50) of the form $U=\frac{1}{18}\left(W(z)-A^{\prime}(t)\left(z^{3}+1\right)\right) A(t)^{-1}$, where $W$ is a solution to ODE (19) with $\alpha=3$. Hence solutions (20), (24), (25) of (19) generate the following solutions of dVN:

$$
\begin{aligned}
& u=\frac{1}{A(t)}\left(\left(y+z_{0} x\right)^{3}-\frac{A^{\prime}(t)}{18}\left(x^{3}+y^{3}\right)\right), \quad z_{0}\left(z_{0}^{3}+1\right)=0 \\
& u=\frac{1}{A(t)}\left(x^{\frac{3}{2}} y^{\frac{3}{2}}-\frac{A^{\prime}(t)}{18}\left(x^{3}+y^{3}\right)\right)
\end{aligned}
$$

and

$$
u=\frac{\left(c_{2}^{3}-2 c_{1}^{3}\right) y^{3}+9 c_{1} c_{2}^{2} x y\left(c_{2} y+c_{1} x\right)+\left(c_{1}^{3}-2 c_{2}^{3}\right) x^{3}-A^{\prime}(t)\left(x^{3}+y^{3}\right)}{A(t)}
$$

with $c_{1} c_{2} \neq 0$. 


\subsection{Reduction of dVN w.r.t. $\chi_{3}$}

For $\chi_{3}$-invariant solutions we have $u_{x}+A u_{y}+\frac{1}{2} A^{\prime} y^{2}=0$, thus such solutions have the form

$$
u=W(t, z)-\frac{A^{\prime}(t) y^{3}}{6 A(t)}, \quad z=y-A(t) x .
$$

Substituting this into (1) and denoting $V=W_{z z}$, we get the nonlinear PDE of first order

$$
V_{t}+\left(2\left(A^{3}-1\right) V+\frac{A^{\prime}}{A} z\right) V_{z}+\frac{2 A^{\prime}}{A} V=0 .
$$

We did not find the general solution to (55), instead we obtain three families of particular solutions for this PDE.

First, we note that substitution $V=-\frac{1}{2} A^{\prime} A^{-1}\left(A^{3}-1\right)^{-1} z$ reduces (55) to the ODE $A^{\prime \prime}=\left(2 A^{3}+1\right)\left(A^{\prime}\right)^{2} A^{-1}\left(A^{3}-1\right)^{-1}$ for function $A$. Integrating this when $A \neq$ const, we get solution of $\mathrm{dVN}$ of the form

$$
u=-\frac{1}{12} \frac{A^{\prime}}{A\left(A^{3}-1\right)}(y-A x)^{3}-\frac{1}{6} \frac{A^{\prime}}{A} y^{3},
$$

where function $A(t)$ is defined implicitly by equation

$$
\ln \frac{(A-1)^{2}}{A^{2}+A+1}+2 \sqrt{3} \arctan \left(\frac{\sqrt{3}}{3}(2 A+1)\right)=c_{1} t+c_{0},
$$

and $c_{0}, c_{1} \neq$ are constants.

When $A(t) \equiv \varepsilon \in \mathbb{R} \backslash\{1\}$, equation (55) gets the form $V_{t}+2\left(\varepsilon^{3}-1\right) V V_{z}=0$. The (multi-valued) solutions of this equation have the form $2\left(\varepsilon^{3}-1\right) t V+G(V)=z$, where $G$ is an arbitrary function of one variable. They produce the family of solutions

$$
u=W(t, z), \quad z=y-\varepsilon x,
$$

where function $W$ is defined implicitly by ODE

$$
2\left(\varepsilon^{3}-1\right) t W_{z z}+G\left(W_{z z}\right)=z .
$$

When $A(t) \equiv 1$, we have $W_{t z z}=0$, and hence

$$
u=H(y-x)+p_{1}(t)(y-x)+p_{0}(t)
$$

with arbitrary functions $H, p_{0}, p_{1}$ of one variable. We can put $p_{0} \equiv p_{1} \equiv 0$ without loss generality in accordance with Remark 3. 


\subsection{Reduction of dVN w.r.t. $\chi_{4}$}

Solutions of $\mathrm{dVN}$ that are invariant with respect to $\chi_{4}$ satisfy equation $u_{y}=A(t) x$ and therefore they have the form $u=A(t) x y+W(t, x)$. When $A \neq 0$, substituting this into (1) gives $W_{x x x}=A^{\prime} A^{-1}$, so we have solutions of $\mathrm{dVN}$ in the form

$$
u=\frac{1}{6} \frac{A^{\prime}(t)}{A(t)} x^{3}+A(t) x y+p_{2}(t) x^{2}
$$

with arbitrary function $p_{2}$.

Finally, for $A \equiv 0$, equation $\mathrm{dVN}$ is satisfied identically, so

$$
u=W(t, x)
$$

is a solution to $\mathrm{dVN}$ for arbitrary function $W$ of two variables.

\section{Non-invariant solutions}

Some of the solutions obtained in Sect. 4 admit natural generalizations that are not invariant with respect to symmetries from $\operatorname{Sym}_{0}(\mathrm{dVN})$.

For example, solution (62) is a particular case of the family of solutions $u=$ $f(t, x)+g(t, y)$ with arbitrary functions $f$ and $g$. The $\sigma_{2}-$ invariant solutions from subsection 4.1.2.2 are included is the set of separable solutions of the form $u=$ $X_{\alpha}(x) Y_{-\alpha}(y)$, where $X_{\alpha}$ and $Y_{\alpha}$ are independently defined by either one of systems (33)-(34), (36)-(37), (40)-(41), or equation (38), or (39).

Nontrivial polynomial solutions (11), (14), (18), (27), (28), (51), (53), (56)-(57), (61) lead to idea to consider solutions of the form

$$
u=\sum_{1 \leq i+j \leq N} T_{i j}(t) x^{i} y^{j}
$$

Below we present such solutions for $N \geq 3$.

\section{$5.1 N=3$}

Substituting (63) into (1) yields system of ODEs

$$
\left\{\begin{array}{l}
T_{21}^{\prime}=6\left(2 T_{30}+T_{03}\right) T_{21}+2 T_{12}^{2} \\
T_{12}^{\prime}=6\left(T_{30}+2 T_{03}\right) T_{12}+2 T_{21}^{2} \\
T_{11}^{\prime}=6\left(T_{30}+T_{03}\right) T_{11}+4\left(T_{20} T_{21}+T_{02} T_{12}\right)
\end{array}\right.
$$

for unknown functions $T_{21}, T_{12}, T_{11}$ and arbitrary functions $T_{30}, T_{03}, T_{20}, T_{02}$ of $t$.

Solutions (11), (14), (18), (27), (51), (53), (56)-(57), (61) are particular cases of solutions given by system (63)-(64) for appropriate choices of functions $T_{30}, T_{03}, T_{20}$, $T_{02}$. 
Analysis of system (64) gives two families of solutions to dVN. The first one reads

$$
\begin{aligned}
u= & -\frac{1}{18}\left(\frac{T_{21}^{\prime}}{T_{21}}\left(y^{3}-2 x^{3}+\frac{3}{2} \frac{T_{11}}{T_{21}} x^{2}\right)+\frac{T_{12}^{\prime}}{T_{12}}\left(x^{3}-2 y^{3}+\frac{3}{2} \frac{T_{11}}{T_{21}} x^{2}\right)\right) \\
& +\frac{1}{4} \frac{T_{11}^{\prime}}{T_{11}} x^{2}+T_{02} y^{2}+\frac{1}{9 T_{21} T_{12}}\left(\left(T_{21}^{3}-2 T_{12}^{3}\right) x^{3}+\left(T_{12}^{3}-2 T_{21}^{3}\right) y^{3}\right) \\
& +x y\left(T_{21} x+T_{12} y+T_{11}\right),
\end{aligned}
$$

where $T_{21}, T_{12}, T_{11}$, and $T_{02}$ are arbitrary functions of $t$ such that $T_{21} \neq 0$ and $T_{12} \neq 0$. When $T_{21} \equiv T_{12} \equiv 0$, we obtain solution

$$
u=T_{30}\left(x^{3}-y^{3}\right)+\frac{1}{6} \frac{T_{11}^{\prime}}{T_{11}} y^{3}+T_{20} x^{2}+T_{11} x y+T_{02} y^{2}
$$

with arbitrary functions $T_{11}, T_{30}, T_{20}, T_{02}$ of $t$ such that $T_{11} \neq 0$.

\section{$5.2 N=4$}

Substituting (63) with $N=4$ into (1) and analyzing the resulting system we get four families of solutions of $\mathrm{dVN}$ :

$$
\begin{aligned}
& u=T_{1}\left(4 x^{3} y-3 y^{4}\right)+\frac{T_{1}^{\prime}}{6 T_{1}} y^{3}+T_{2} y^{2}, \quad T_{1} \neq 0, \\
& u=T\left(17 x^{4}-36 x^{3} y-90 x^{2} y^{2}-36 x y^{3}+17 y^{4}\right)+\frac{1}{6} \frac{T^{\prime}}{T}\left(x^{3}+y^{3}\right), \\
& T \neq 0 \text {, } \\
& u=T\left(17 x^{4}-36 x^{3} y-90 x^{2} y^{2}-36 x y^{3}+17 y^{4}\right)+\frac{1}{6} \frac{T^{\prime}}{T}\left(x^{3}+y^{3}\right), \\
& T \neq 0, \quad c \in \mathbb{R}, \\
& u=\frac{4 T^{2} T^{\prime} T^{\prime \prime \prime}-8 T^{2}\left(T^{\prime \prime}\right)^{2}+3\left(T^{\prime}\right)^{4}}{1728 T^{2}\left(T^{\prime}\right)^{16 / 3}}\left(2 T T^{\prime \prime}\left(x^{2}+y^{2}\right)-3\left(T^{\prime}\right)^{2} y^{2}\right) \\
& +\left(T^{\prime}\right)^{4 / 3}(x-y)^{4}-\frac{1}{18} \frac{T^{\prime \prime}}{T^{\prime}} y\left(3 x^{2}-3 x y+2 y^{2}\right) \\
& +\frac{1}{12} \frac{T^{\prime}}{T} y\left(3 x^{2}+y^{2}\right), \quad T \neq 0, T^{\prime} \neq 0
\end{aligned}
$$

Solution (68) with $T \equiv 1$ coincides with solution (28).

\section{$5.3 N=5$}

For $N=5$ we obtain the family of solutions

$$
u=T_{50}(x-y)^{5}+T_{30} x\left(x^{2}+3 y^{2}\right)+T_{03} y\left(y^{2}+3 x^{2}\right)
$$




$$
+\frac{1}{30} \frac{T_{50}^{\prime}}{T_{50}} y^{2}(y-3 x)+T_{11} x y,
$$

where $T_{50}$ is an arbitrary nonzero function of $t$, while $T_{30}, T_{03}$, and $T_{11}$ satisfy the following system of ODEs:

$$
\left\{\begin{aligned}
T_{30}^{\prime}= & \frac{1}{150}\left(\frac{5 T_{50}^{\prime \prime}}{T_{50}}-\frac{7\left(T_{50}^{\prime}\right)^{2}}{T_{50}^{2}}+\frac{30 T_{50}^{\prime}}{T_{50}}\right)\left(T_{30}-2 T_{03}\right) \\
& +6\left(T_{30}+T_{03}\right)^{2} \\
T_{03}^{\prime}= & \frac{\left(T_{50}^{\prime}\right)^{2}}{150 T_{50}}+\frac{T_{50}^{\prime}}{5 T_{50}}\left(T_{03}-2 T_{30}\right)+6\left(T_{30}+T_{03}\right)^{2} \\
T_{11}^{\prime}= & \left(\frac{T_{50}^{\prime}}{5 T_{50}}+6\left(T_{30}+T_{03}\right)\right) T_{11}
\end{aligned}\right.
$$

\section{$5.4 N \geq 6$}

For each $N \geq 6$ we find the family of solutions

$$
\begin{aligned}
u= & \sum_{k=4}^{N} T_{k}(x-y)^{k}+T_{30}\left(x^{3}+3 x^{2} y\right)+T_{03}\left(y^{3}+3 x y^{2}\right) \\
& +\frac{1}{2 N^{2}} \frac{T_{N}^{\prime}}{T_{N}} y\left((N-1) T_{N-1} y-N T_{N} x(x+y)\right) \\
& -\frac{1}{2 N} \frac{T_{N-1}^{\prime}}{T_{N}} y^{2}+T_{20}\left(x^{2}+y^{2}\right)+T_{11} x y,
\end{aligned}
$$

where $T_{N}, T_{N-1}$, and $T_{20}$ are arbitrary functions of $t$ such that $T_{N} \neq 0, T_{N-1} \neq 0$, while functions $T_{k}$ with $k \in\{4, \ldots N-2\}, T_{30}, T_{03}$, and $T_{11}$ obey the following system of ODEs:

$$
\left\{\begin{aligned}
T_{k}^{\prime}= & \frac{1}{N^{2} T_{N}^{2}}\left(\left(k N T_{k} T_{N}-(k+1)(N-1) T_{k+1} T_{N-1}\right) T_{N}^{\prime}\right. \\
& \left.+(k+1) N T_{k+1} T_{N} T_{N-1}^{\prime}\right), \quad k \in\{4, \ldots, N-2\}, \\
T_{30}^{\prime}= & \frac{1}{6 N^{2} T_{N}^{2}}\left(N T_{N} T_{N}^{\prime \prime}-(N-1)\left(T_{N}^{\prime}\right)^{2}\right. \\
& -24 T_{4}\left((N-1) T_{N-1} T_{N}^{\prime}-N T_{N} T_{N-1}^{\prime}\right) \\
& \left.+36 N^{2} T_{N}^{2}\left(T_{30}+T_{03}\right)^{2}-6 N\left(T_{30}+4 T_{03}\right) T_{N} T_{N}^{\prime}\right), \\
T_{03}^{\prime}= & \frac{1}{6 N^{2} T_{N}^{2}}\left(N T_{N} T_{N}^{\prime \prime}-(N-1)\left(T_{N}^{\prime}\right)^{2}\right. \\
& -24 T_{4}\left((N-1) T_{N-1} T_{N}^{\prime}-N T_{N} T_{N-1}^{\prime}\right) \\
& \left.+36 N^{2} T_{N}^{2}\left(T_{30}+T_{03}\right)^{2}-6 N\left(4 T_{30}+T_{03}\right) T_{N} T_{N}^{\prime}\right), \\
T_{11}^{\prime}= & \frac{1}{N^{3} T_{N}^{3}}\left(\left(N T_{N} T_{N-1}^{\prime}-(N-1) T_{N-1} T_{N}^{\prime}\right) T_{N}^{\prime}\right. \\
& +6 N^{3} T_{N}^{3}\left(T_{30}+T_{03}\right)\left(T_{11}+2 T_{20}\right)-6 N^{2} T_{N}^{2} T_{30} T_{N-1}^{\prime} \\
& \left.+6 N\left((N-1) T_{N} T_{30}-\frac{2}{3} N T_{N} T_{20}\right) T_{N} T_{N}^{\prime}\right) .
\end{aligned}\right.
$$




\section{Cosymmetries and conservation laws}

Cosymmetries of equation (1) are solutions $\psi$ to equation (4), which is of the form

$$
\begin{aligned}
D_{t} D_{x} D_{y}(\psi)=u_{x y} & \left(D_{x}^{3}(\psi)+D_{y}^{3}(\psi)\right)+u_{x x} D_{x}^{2} D_{y}(\psi)+u_{y y} D_{x} D_{y}^{2}(\psi) \\
& +3 u_{x x y} D_{x}^{2}(\psi)+\left(u_{x x x}+u_{y y y}\right) D_{x} D_{y}(\psi)+3 u_{x y y} D_{y}^{2}(\psi) \\
& +2 u_{x x x y} D_{x}(\psi)+2 u_{x y y y} D_{y}(\psi) .
\end{aligned}
$$

Direct computations show that each solution $\psi \in C^{\infty}\left(J^{2}(\pi)\right)$ of equation (75) is a linear combination of the following cosymmetries:

$$
\begin{aligned}
& \psi_{1}= \ln u_{x y}, \\
& \psi_{2}=A \\
& \psi_{3}= 2 A u_{x x}+A^{\prime} x \\
& \psi_{4}= 2 A u_{y y}+A^{\prime} y, \\
& \psi_{5}= 4 A\left(2\left(u_{t x}-u_{x y} u_{y y}\right)+u_{x x}^{2}\right)+2 A^{\prime}\left(2 x u_{x x}+u_{x}\right)+A^{\prime \prime} x^{2}, \\
& \psi_{6}=4 A\left(2\left(u_{t y}-u_{x y} u_{x x}\right)+u_{y y}^{2}\right)+2 A^{\prime}\left(2 y u_{y y}+u_{y}\right)+A^{\prime \prime} y^{2}, \\
& \begin{aligned}
\psi_{7}= & 12 A\left(3 u_{t t}+6\left(u_{t x} u_{x x}+u_{t y} u_{y y}\right)-18 u_{x x} u_{x y} u_{y y}-2 u_{x y}^{3}\right) \\
& \quad-6 A^{\prime}\left(3 u_{t}+4\left(x\left(u_{t x}-u_{x y} u_{y y}\right)+y\left(u_{t y}-u_{x y} u_{x x}\right)\right)\right. \\
& \left.\quad+2\left(u_{x x}\left(x u_{x x}+u_{x}\right)+u_{y y}\left(y u_{y y}+u_{y}\right)\right)\right)-A^{\prime \prime \prime}\left(x^{3}+y^{3}\right) \\
& \quad-6 A^{\prime \prime}\left(\left(x u_{x}+y u_{y}+y^{2} u_{y y}+x^{2} u_{x x}\right) .\right.
\end{aligned}
\end{aligned}
$$

Here $A=A(t)$ are arbitrary (smooth) functions.

The conservation laws $\Omega_{1}, \ldots, \Omega_{7}$ associated to cosymmetries $\psi_{1}, \ldots, \psi_{7}$ are given by the formulas

$$
\begin{aligned}
\Omega_{1}= & u_{x y}\left(\ln u_{x y}-1\right) d x \wedge d y+\left(\frac{1}{2} u_{y y}^{2}-u_{x x} u_{x y} \ln u_{x y}\right) d y \wedge d t \\
+ & \left(\frac{1}{2} u_{x x}^{2}-u_{x y} u_{y y} \ln u_{x y}\right) d t \wedge d x \\
\Omega_{2} & =2 A u_{x y}\left(d x \wedge d y-u_{x x} d y \wedge d t-u_{y y} d x \wedge d t\right)-A^{\prime}\left(u_{y} d y-u_{x} d x\right) \wedge d t, \\
\Omega_{3} & =A\left(3 u_{x x} u_{x y} d x \wedge d y-3 u_{x y}\left(u_{t x}+u_{x x}^{2}+u_{x y} u_{y y}\right) d y \wedge d t\right. \\
& \left.+\left(3 u_{x x}\left(u_{t x}-2 u_{x y} u_{y y}\right)+u_{x x}^{3}+u_{x y}^{3}\right) d t \wedge d x\right) \\
& -3 A^{\prime} x\left(u_{x x} u_{x y} d y+\left(u_{t x}-u_{x y} u_{y y}\right) d x\right) \wedge d t, \\
\Omega_{4} & =A\left(3 u_{y y} u_{x y} d x \wedge d y-3 u_{x y}\left(u_{t y}+u_{y y}^{2}+u_{x y} u_{x x}\right) d x \wedge d y\right. \\
& \left.+\left(3 u_{y y}\left(u_{t y}-2 u_{x y} u_{x x}\right)+u_{y y}^{3}+u_{x y}^{3}\right) d y \wedge d t\right) \\
& +3 A^{\prime} y\left(u_{y y} u_{x y} d x+\left(u_{t y}-u_{x y} u_{x x}\right) d y\right) \wedge d t, \\
\Omega_{5} & =u_{x y}\left(4 A u_{x x}^{2}+2 A^{\prime} x u_{x x}+A^{\prime \prime} x^{2}\right) d x \wedge d y
\end{aligned}
$$




$$
\begin{aligned}
& +\left(A\left(8 u_{x y} u_{x x}\left(u_{y y} u_{x y}-u_{t x}\right)-\frac{2}{3} u_{x y}\left(u_{x x}^{3}-u_{x y}^{3}\right)\right)\right. \\
& \left.+2 A^{\prime}\left(x u_{x y}\left(u_{x y} u_{y y}-u_{t x}-u_{x x}^{2}\right)-u_{x} u_{x x} u_{x y}\right)-A^{\prime \prime} x^{2} u_{x x} u_{x y}\right) d y \wedge d t \\
& +\left(A \left(4\left(\left(u_{t x}-u_{y y} u_{x y}\right)^{2}-u_{x y} u_{y y} u_{x x}^{2}\right)-\frac{2}{3} u_{x x}\left(4 u_{x y}^{3}+u_{x x}^{3}\right)\right.\right. \\
& +A^{\prime}\left(2 u_{t x}\left(x u_{x x}+u_{x}\right)-2 u_{y y} u_{x y}\left(2 x u_{x x}+u_{x}\right)-\frac{2}{3} x\left(u_{x y}^{3}+u_{x x}^{3}\right)\right) \\
& \left.-A^{\prime \prime} x^{2} u_{x y} u_{y y}-A^{\prime \prime \prime} x^{2} u_{x}\right) d t \wedge d x \\
& \Omega_{6}=u_{x y}\left(4 A u_{y y}^{2}+2 A^{\prime} y u_{y y}+A^{\prime \prime} y^{2}\right) d x \wedge d y \\
& +\left(A \left(4\left(\left(u_{t y}-u_{x x} u_{x y}\right)^{2}-u_{x x} u_{x y} u_{y y}^{2}\right)-\frac{2}{3} u_{y y}\left(4 u_{x y}^{3}+u_{y y}^{3}\right)\right.\right. \\
& +A^{\prime}\left(2 u_{t y}\left(y u_{y y}+u_{y}\right)-2 u_{x x} u_{x y}\left(2 y u_{y y}+u_{y}\right)-\frac{2}{3} y\left(u_{x y}^{3}+u_{y y}^{3}\right)\right) \\
& \left.-A^{\prime \prime} y^{2} u_{x x} u_{x y}-A^{\prime \prime \prime} y^{2} u_{y}\right) d y \wedge d t \\
& +\left(A\left(8 u_{x y} u_{y y}\left(u_{x x} u_{x y}-u_{t y}\right)-\frac{2}{3} u_{x y}\left(u_{y y}^{3}-u_{x y}^{3}\right)\right)\right. \\
& \left.+2 A^{\prime}\left(y u_{x y}\left(u_{x x} u_{x y}-u_{t y}-u_{y y}^{2}\right)-u_{y} u_{x y} u_{y y}\right)-A^{\prime \prime} y^{2} u_{x y} u_{y y}\right) d t \wedge d x, \\
& \Omega_{7}=\sum_{k=0}^{3} \frac{d^{k} A}{d t^{k}}\left(P_{k} d x \wedge d y+Q_{k} d y \wedge d t+R_{k} d t \wedge d x\right)
\end{aligned}
$$

where

$$
\begin{aligned}
& P_{0}=\frac{1}{12}\left(2 u_{x y}\left(6\left(u_{t x} u_{x x}+u_{t y} u_{y y}\right)+u_{x x}^{3}-3 u_{x x} u_{x y} u_{y y}+u_{y y}^{3}\right)-3 u_{t x} u_{t y}\right), \\
& P_{1}=\frac{1}{12} u_{x y}\left(u_{x} u_{x x}+u_{y} u_{y y}+3\left(y u_{t y}+u_{t}\right)+2\left(x u_{x x}^{2}+y u_{y y}^{2}\right)-3 y u_{t x} u_{y y}\right) \text {, } \\
& P_{2}=\frac{1}{24}\left(6 t\left(\left(x u_{x y}+y u_{y y}\right) u_{t x}+u_{x y}\left(x^{2} u_{x x}+y^{2} u_{y y}+2\left(x u_{x}+y u_{y}\right)\right)\right)\right. \\
& \left.-\left(x u_{x x}+y u_{x y}\right) u_{t y}\right) \\
& Q_{0}=\frac{1}{12}\left(3 u_{t y}^{2} u_{y y}+u_{x y}\left(6 u_{x y} u_{y y}\left(6 u_{t x}+3 u_{x x}^{2}-u_{x y} u_{y y}\right)-u_{t x}\left(u_{t x}+6 u_{x x}^{2}\right)\right)\right. \\
& \left.+\left(3 u_{t t}-12 u_{x x} u_{x y} u_{y y}-2 u_{y y}^{3}-2 u_{x y}^{3}\right) u_{t y}+2 u_{x y}\left(2 u_{x y}^{3}-3 u_{t t}\right) u_{x x}\right), \\
& Q_{1}=\frac{1}{36}\left(u_{x y}^{3}\left(x u_{x y}-u_{y}\right)-3 u_{x y}\left(4 x u_{x x}+3 u_{x}\right) u_{t x}-3 y u_{t y}^{2}-2 x u_{x x}^{3} u_{x y}\right. \\
& -3\left(4 y u_{x x} u_{x y}-u_{y} u_{y y}\right) u_{t y}+3 u_{x y}\left(2 y u_{x y}-u_{x}\right) u_{x x}^{2}-u_{y y}^{3}\left(y u_{y y}+u_{y}\right) \\
& -3 u_{x y}\left(3 u_{t}+2 y u_{y y}^{2}-2\left(2 x u_{x y}-u_{y}\right) u_{y y}\right) u_{x x} \\
& \left.+\left(3 u_{x} u_{x y}^{2}+9 y u_{t t}-4 y u_{x y}^{3}\right) u_{y y}\right), \\
& Q_{2}=\frac{1}{72}\left(3\left(y^{2} u_{t y} u_{y y}+6 t\left(y u_{t y}^{2}+x u_{t t} u_{x y}\right)-\left(x^{2} u_{x y}+6 t x u_{t y}\right) u_{t x}\right)\right. \\
& -3\left(x^{2} u_{x y} u_{x x}^{2}+2 u_{x y}\left(x u_{x}+y u_{y}+y^{2} u_{y y}\right) u_{x x}+\left(6 t y u_{t t}-x^{2} u_{x y}^{2}\right) u_{y y}\right) \\
& \left.-y^{2}\left(u_{x y}^{3}+u_{y y}^{3}\right)-18 x u_{t} u_{x y}\right) \text {, }
\end{aligned}
$$




$$
\begin{aligned}
Q_{3}=\frac{1}{72} & \left(\left(18 t\left(x u_{x}+y u_{y}-u\right)+y^{3}\right) u_{t y}-\left(x^{3}+y^{3}\right) u_{x x} u_{x x}-3 y u_{y}^{2}\right), \\
R_{0}=\frac{1}{12} & \left(3\left(u_{t x}^{2} u_{x x}-u_{x y} u_{t y}^{2}\right)+\left(3 u_{t t}-12 u_{x x} u_{x y} u_{y y}-2\left(u_{x x}^{3}+u_{x y}^{3}\right)\right) u_{t x}\right. \\
& -6\left(u_{x x}^{2} u_{x y}^{3}-u_{x y}\left(u_{x x} u_{x y}-u_{y y}^{2}\right) u_{t y}-3 u_{x x} u_{x y}^{2} u_{y y}^{2}\right) \\
& \left.+2 u_{x y}\left(4 u_{x y}^{4}-3 u_{t t}\right) u_{y y}\right),
\end{aligned}
$$

$$
\begin{aligned}
R_{1}=\frac{1}{36} & \left(3\left(2 x u_{t x}^{2}+\left(3 y u_{t y}-4 x u_{x y} u_{y y}+2 u_{x} u_{x x}\right) u_{t x}\right)-u_{x x}^{3}\left(x u_{x x}+u_{x}\right)\right. \\
& -3 u_{x y}\left(\left(4 y u_{y y}+3 u_{y}\right) u_{t y}-\left(2 x u_{x y}-u_{y}\right) u_{y y}^{2}+3\left(y u_{t t}-u_{t} u_{y y}\right)\right) \\
& +2 u_{x y} u_{y y}\left(\left(2 y u_{x y}-u_{x}\right)-3 x u_{x x}^{2} u_{y y}-y u_{y y}^{2}\right)+u_{x y}^{3}\left(y u_{x y}-u_{x}\right) \\
& \left.+u_{x x} u_{x y}^{2}\left(3 u_{y}-4 x u_{x y}\right)\right), \\
R_{2}=\frac{1}{72}( & 18 t\left(y u_{t t} u_{x y}-x u_{t x}^{2}\right)+\left(3 x^{2} u_{x x}-18 t y u_{t y}\right) u_{t x}-x^{2}\left(u_{x x}^{3}+u_{x y}^{3}\right) \\
& -3\left(y^{2} u_{x y}\left(u_{t y}+u_{y y}^{2}\right)-\left(6 x\left(t u_{t t}+u_{t}\right)-u_{x y}\left(2 x^{2} u_{y y}+y^{2} u_{x y}\right)\right) u_{x x}\right) \\
& \left.-6 u_{x y}\left(x u_{x}+y u_{y}\right) u_{y y}\right), \\
R_{3}=\frac{1}{72}( & \left.\left(18 t\left(u-x u_{x}-y u_{y}\right)+x^{3}\right) u_{t x}-\left(x^{3}+y^{3}\right) u_{x y} u_{y y}-3 x u_{x}^{2}\right) .
\end{aligned}
$$

\section{Concluding remarks}

The results of the paper can be summarized as follows. Employing the methods of the Lie symmetry group analysis we have found a number of exact solutions for the dispersionless Veselov-Novikov equation, including solutions in elementary or elliptic functions (17), (33)-(34), (36)-(37), (38), (39), (40)-(41), (52), or functions represented by quadratures (15)-(16), (22)-(23), (58)-(59). We have indicated ordinary differential equations (10), (19), (43), (45), (47), (49) that describe all other invariant solutions. We have studied some non-invariant solutions and found a broad set of polynomial solutions (65)—(74). Furthermore, we have presented all the local conservation laws of order up to two.

While ODEs (10), (19), (43), (45), (47), (49) are not integrable by quadratures in general, their origin as reductions of Lax-integrable PDEs (8) and (50) allows one to hope that the method of prolongation structures in the version implemented in [16] could be applicable to examine these ODEs. Likewise, the methods of weak symmetries [19,20], nonclassical symmetry reductions, see [5] and references therein, conditional symmetries [7,8], or stable-range approach [26], can be useful to generate new non-invariant solutions of the dispersionless Veselov-Novikov equation. We intend to address these issues in our future work.

Acknowledgements The work of O.M. was partially supported by the Faculty of Applied Mathematics of AGH UST statutory tasks within subsidy of Ministry of Science and Higher Education (Poland). 
The work of J.-H.C. was partially supported by the Ministry of Science and Technology (Taiwan), research project number MOST 109-2115-M-606-001.

The authors are very grateful to M.V. Pavlov for stimulating discussions and important comments.

Data Availability The authors confirm that the data supporting the findings of this study are available within the article.

\section{Declarations}

Conflict of interest The authors declare that they have no conflict of interest.

Ethical approval The authors declare that they have adhered to the ethical standards of research execution.

Open Access This article is licensed under a Creative Commons Attribution 4.0 International License, which permits use, sharing, adaptation, distribution and reproduction in any medium or format, as long as you give appropriate credit to the original author(s) and the source, provide a link to the Creative Commons licence, and indicate if changes were made. The images or other third party material in this article are included in the article's Creative Commons licence, unless indicated otherwise in a credit line to the material. If material is not included in the article's Creative Commons licence and your intended use is not permitted by statutory regulation or exceeds the permitted use, you will need to obtain permission directly from the copyright holder. To view a copy of this licence, visit http://creativecommons.org/licenses/by/4.0/.

\section{References}

1. Baran, H., Marvan, M.: Jets: A software for differential calculus on jet spaces and diffieties. Available on-line at http://jets.math.slu.cz

2. Bogdanov, L.V., Konopelchenko, B.G., Moro, A.: Symmetry constraints for real dispersionless Veselov-Novikov equation. J. Math. Sci. 136(6), 4411-4418 (2006)

3. Chebyshev, P.L.: (Tchebychef). Sur l'intégration des différentielles irrationelles. Journal de mathématiques pures at appliquées. I. serie, XVIII (1853), 87-111, Euvres T. I, pp. 142-168, Reprint. Chelsea, N.Y., (1961)

4. Chebyshev, P.L.: (Tchebychef). Sur l'intégration des différentielles les plus simples parmi celles qui contiennent une racine cubique. Matem. Sbornik II (1867), 71-78, Euvres T. II, pp. 41-47, Reprint. Chelsea, N.Y., (1961)

5. Clarkson, P.A.: Nonclassical symmetry reductions of nonlinear partial differential equations. Math. Comput. Model. 18, 45-68 (1993)

6. Corless, R.M., Gonnet, G.H., Hare, D.E.G., Jeffrey, D.J., Knuth, D.E.: On the Lambert W function. Adv. Comput. Math. 5, 329-359 (1996)

7. Fushchich, W.I., Shtelen, W.M., Serov, N.I.: Symmetry Analysis and Exact Solutions of the Equations of Mathematical Physics. Kluwer, Dordrecht (1993)

8. Fushchich, W., Tsyfra, I.: On reduction and solutions of nonlinear wave equation with broked symmetry. J. Phys. A, Math. Gen. 20, 45-60 (1987)

9. Golubev, V.V.: P.L. Chebyshev's works on integration of algebraic functions. Scientific Heritage of P.L. Chebyshev. First Issue, Mathematics. Academy of Science Publishing, Moscow, 1945, pp. 88-121 (in Russian)

10. Hardy, G.H.: The integration of Functions of a Single Variable, 2nd edn. Cambridge University Press, Cambridge (1916)

11. Konopelchenko, B., Martínez Alonso, L.: Nonlinear dynamics on the plane and integrable hierarchies of infinitesimal deformations. Stud. Appl. Math. 109, 313-336 (2002)

12. Konopelchenko, B., Moro, A.: Integrable equations in nonlinear geometrical optics. Stud. Appl. Math. 113, 325-352 (2004)

13. Krasil'shchik, J., Verbovetsky, A.: Geometry of jet spaces and integrable systems. J. Geom. Phys. 61, 1633-1674 (2011) 
14. Krasil'shchik, J., Verbovetsky, A., Vitolo, R.: The Symbolic Computation of Integrability Structures for Partial Differential Equations. Springer, Berlin (2017)

15. Krasil'shchik, I.S., Vinogradov, A.M.: Nonlocal trends in the geometry of differential equations: symmetries, conservation laws, and Bäcklund transformations. Acta Appl. Math. 15, 161-209 (1989)

16. Morris, H.C., Dodd, R.K.: Infinite dimensional Lie algebras and the direct determination of deformation problems for equations of Painlevé type. Proc. Roy. Irish Acad. 83A, 127-143 (1983)

17. Nizhnik, L.P.: Integration of multidimensional nonlinear equations by the method of the inverse problem. Sov. Phys. Dokl. 25, 706-708 (1981)

18. Olver, P.J.: Applications of Lie Groups to Differential Equations, 2nd edn. Springer, Berlin, New York, Heidelberg (2000)

19. Olver, P.J., Rosenau, Ph: The construction of special solutions to partial differential equations. Phys. Lett. A 114, 107-112 (1986)

20. Olver, P.J., Vorob'ev, E.M.: Nonclassical conditions symmetries of partial differential equations. CRC Handbook of Lie Group Analysis of Differential Equations, V.3, Modern Trends, CRC Press, Boca Raton, pp. 291-328 (1996)

21. Pavlov, M.V.: Modified dispersionless Veselov-Novikov equation and corresponding hydrodynamic chains. arXiv:nlin/0611022

22. Ritt, J.F.: Integration in Finite Terms. Liouville's Theory of Elementary Methods. Columbia University Press, New York (1948)

23. Taimanov, I.A.: On first integrals of geodesic flows on a two-torus. Proc. Steklov Inst. Math. 275, 225-242 (2016)

24. Veselov, A.P., Novikov, S.P.: Finite-zone two-dimensional potential Schrödinger operators. Explicit formulas and evolution equations. Sov. Math. Dokl. 30, 588-591 (1984)

25. Vinogradov, A.M., Krasil'shchik, I.S. (eds.): Symmetries and Conservation Laws for Differential Equations of Mathematical Physics [in Russian], Moscow: Factorial, 2005; English transl. prev. ed.: I.S. Krasil'shchik, A.M. Vinogradov (eds.) Symmetries and Conservation Laws for Differential Equations of Mathematical Physics. Transl. Math. Monogr., 182, Amer. Math. Soc., Providence, RI, (1999)

26. Xiaoping, Xu: Algebraic Approaches to Partial Differential Equations. Springer, Berlin (2013)

Publisher's Note Springer Nature remains neutral with regard to jurisdictional claims in published maps and institutional affiliations. 